\title{
The Effect of The Forward Head Posture on Postural Balance in Long Time Computer Based Worker
}

\author{
Jung-Ho Kang, M.D., Rae-Young Park, M.D., Su-Jin Lee, M.D., Ja-Young Kim, M.D., \\ Seo-Ra Yoon, M.D., Kwang-Ik Jung, M.D.
}

Department of Rehabilitation Medicine, Gwangju Veterans Hospital, Gwangju 506-705, Korea

\begin{abstract}
Objective To estimate the effects of a relatively protruded head and neck posture on postural balance, in computer based worker.

Method Thirty participants, who work with computers for over 6 hrs per day (Group I), and thirty participants, who rarely work with computers (Group II), were enrolled. The head and neck posture was measured by estimating angles A and B. A being the angle between the tragus of the ear, the lateral canthus of the eye, and horizontal line and $\mathrm{B}$ the angle between the $\mathrm{C} 7$ spinous process, the tragus of the ear, and the horizontal line. The severity of head protrusion with neck extension was assessed by the subtraction of angle A from angle B. We also measured the center of gravity (COG) and postural balance by using computerized dynamic posturography to determine the effect of computer-based work on postural balance.

Results Results indicated that group I had a relatively more protruded head with extensive neck posture (angle B-A of group I and group II, 28.2 $\pm 8.3,32.9 \pm 6.0 ; \mathrm{p}<.05$ ). The COG of group I tended more toward the anterior than that of group II. Postural imbalance and impaired ability to regulate movement in forward and backward direction were also found.

Conclusion The results of this study suggest that forward head postures during computer-based work may contribute to some disturbance in the balance of healthy adults. These results could be applied to education programs regarding correct postures when working at a computer for extended periods of time.
\end{abstract}

Key Words Turtle neck, Center of gravity, Postural balance

Received February 22, 2011; Accepted October 27, 2011

Corresponding author: Seo-Ra Yoon

Department of Rehabilitation Medicine, Gwangju Veterans Hospital, Sanwol-dong, Gwangsan-gu, Gwangju 506-705, Korea

Tel: +82-62-602-6295, Fax: +82-62-602-6990,E-mail: standupmd@ hanmail.net

(c) This is an open-access article distributed under the terms of the Creative Commons Attribution Non-Commercial License (http:// creativecommons.org/licenses/by-nc/3.0) which permits unrestricted noncommercial use, distribution, and reproduction in any medium, provided the original work is properly cited.

Copyright () 2012 by Korean Academy of Rehabilitation Medicine

\section{INTRODUCTION}

These days, processing a large amount of data, and maintaining a global database using a a computer (commonly called visual display terminal (VDT)) is becoming increasingly popular. ${ }^{1}$ According to the National Statistical Office, with an increasing amount of people in possession of a computer, and an extended internet network, the weekly mean time that a person will spend at a com- 
puter has dramatically increased, from 5.9 hours in 1997 to 14.6 hours in 2003 . Also, $56.2 \%$ of computer users use the machine for 10 hours a week or more. Computers improve work productivity and efficiency, but, on the other hand, using them excessively can result in VDT syndrome, with, complaints of musculoskeletal pains, visual disorders, headache, and other symptoms. Among these complaints, musculoskeletal problems is the most common..$^{2-4}$ Regarding this, the World Health Organization defines work-related musculoskeletal disorders as 'injuries in muscles, tendons, peripheral nerves, and vascular vessels, possibly caused by, precedent to, or worsened by repetitive or continuous use of a certain body part. ${ }^{5}$

Among the effects of using a computer on the musculoskeletal system, keeping a posture of staring at a monitor, located below the height of eyesight, for a long time makes the head move forward, which causes exaggerated anterior curve in the lower cervical vertebrae and exaggerated posterior curve in the upper thoracic vertebrae to maintain balance; this is known as the forward head posture (turtle neck posture) ${ }^{6-9}$ This turtle neck posture are becoming increasingly commonly, as it is becoming more common place to use VDTs in the leaning forward posture, particularly with the popularization of Smart phones.

Szeto et al. $^{8}$ and Moore ${ }^{9}$ stated that maintaining the head forward for long periods of time may cause musculoskeletal disorders such as 'upper crossed syndrome', which involves having reduced lordosis of the lower cervical, in conjunction with kyphosis of the upper thoracic vertebrae. Burgess-Limerick et al. ${ }^{10}$ suggested that such posture causes shortening of muscular fibers around articulation atlantooccipitalis and overstretching of muscles around joints and thus possibly chronic neck pain. In addition, Silva et al. ${ }^{11}$ reported, in a comparative study with people with and without non-traumatic neck pain, that patients complaining of non-traumatic neck pain tend to keep the forward head posture, when compared to people without pain. Griegel-Morris et al. ${ }^{12}$ stated that the forward head posture may affect not only neck but also the thoracic spine and shoulder blade, possibly causing overall imbalance in the musculoskeletal system.

Chester ${ }^{13}$ reported that severe neck pain was associated with decreased balancing ability. Panzer et al. reported that neck trauma or vestibular impairment contributed to abnormal biofeedback, the function of maintaining body balance. Additionally, it might change torque required to maintain posture, which is associated with reduced balance controlling ability. Moreover, Barrett et al.$^{14}$ reported that pains or inflammation reduced sense in joints and thereby abnormal proprioception, which led to posture imbalance.

Previous studies reported that poor working postures with a computer, for long periods of time, are associated with neck pain due to musculoskeletal disorder. ${ }^{5}$ Other studies regarding balance reported that reduced sensation of joints is a major contributor to reducing balancing ability ${ }^{14}$ However, there have been no studies directly evaluating the effects of using computers on balancing ability; studies have evaluated only balancing ability, regarding the neck, mainly in patients complaining of pain. ${ }^{15}$ Also, they have not used objective measures to quantify body sway.

Therefore, this study aims to quantify neck postures of individuals who heavily use computer, to determine whether they actually have the forward head posture with comparison to a control group and to then evaluate balancing ability of heavy computer users under the assumption that they may show decreased body balancing ability, even if they do not complain of pain. The Balance Master $^{\circledR}$ system (NeuroCom ${ }^{\circledR}$, Oregon, USA) that measures body sway in an objective manner and identifies the center of gravity, will be utilized.

\section{MATERIALS AND METHODS}

\section{Study sample}

This study was conducted with normal adults aged between 30 and 40 with no abnormal finding confirmed through physical examination and cervical radiology examination, in our rehabilitation department. Through survey, 30 computer workers who had used a computer for greater than or equal to 6 hours, over the course of 10 years, were identified and allocated to the experimental group (Group I). Another 30 healthy adults, whose daily computer usage totaled only about 1 hour, were used as the control group (Group II), with consideration to age and gender. Individuals with a history of treatment for cervical, thoracic or lumbar disorders, and current conditions of joint pains in ankles, knees, or hips, as well as limited mobility that could affect body balancing ability, were excluded from the study. All participants were fully 


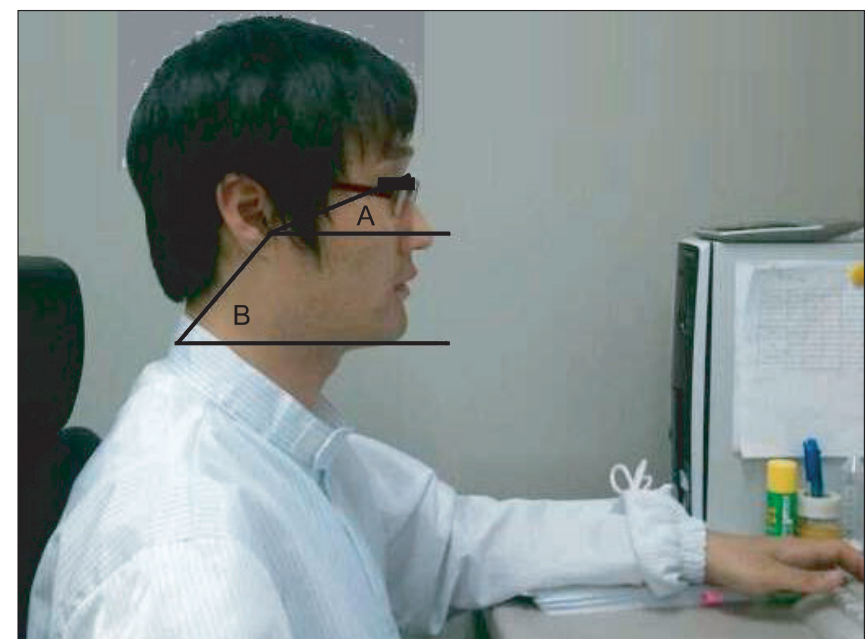

Fig. 1. A is the angle between the tragus of the ear, the lateral canthus of the eye, and the horizontal line of the tragus. B is the angle between $\mathrm{C}$ 7, the tragus of the ear, and the horizontal line of the $\mathrm{C} 7$ spinous process. The severity of the turtle neck posture was assessed by substraction of the angle A and from the angle $\mathrm{B}$.

briefed about the study's purpose, before giving their written consent.

\section{Study method}

To replicate the usual neck posture of office workers using computers, three pictures were taken from their lateral side at 2 hours after using a computer in both experimental and control groups. On the lateral picture taken during their computer operations, the monitorfacing angle (Angle A) was defined by drawing a line from the tragus of ear to the lateral canthus of the eye and the horizontal line of the tragus. The angle of cervical curve (Angle B) was defined as the angle between a line drawn from the spinous process of the $\mathrm{C} 7$ to the tragus and a horizontal line of the $\mathrm{C} 7$ spinous process. The difference, subtracting Angle A from Angle B, was a parameter to quantify severity of forward head posture (Fig. 1). ${ }^{11,16,17}$

Using a foot pressure measurement system, Gait View ${ }^{\circledR}$, subjects' centers of gravity (COG) in both static and dynamic conditions were measured. This was repeated 3 times. The means of the resulting values of COG in both static and dynamic conditions were calculated and compared between groups. ${ }^{18}$

The balancing ability test employed the SMART Balance Master $^{\circledR}$ system for quantification. The test procedures were fully explained to subjects the day before the test. Among assessment programs the of Balance Master ${ }^{\circledR}$
Table 1. Six Conditions of Sensory Organization Test

\begin{tabular}{lcll}
\hline & Force platform & \multicolumn{1}{c}{ Eyes } & \multicolumn{1}{c}{ Screen } \\
\hline Condition 1 & Fixed & Open & Fixed \\
Condition 2 & Fixed & Closed & Fixed \\
Condition 3 & Fixed & Open & Movement \\
Condition 4 & Sway & Open & Fixed \\
Condition 5 & Sway & Closed & Fixed \\
Condition 6 & Sway & Open & Movement \\
\hline
\end{tabular}
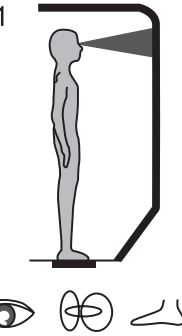

4

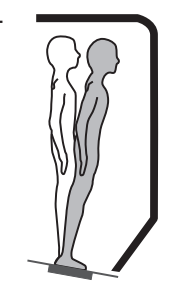

$\infty<$
2

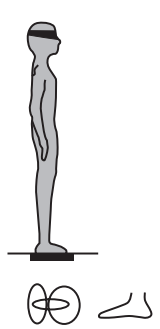

5

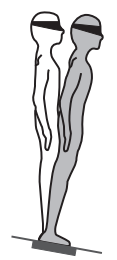

\&)
3

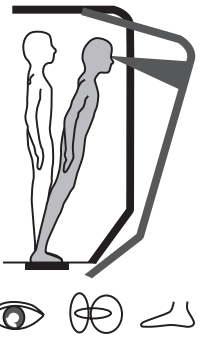

6

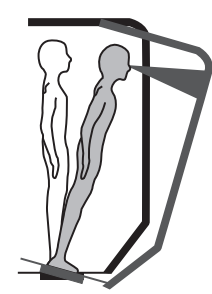

(d) $\theta<$
Sensory oganization test

Fig. 2. Six conditions of sensory organization test (SOT)

system, the Sensory Organization Test (SOT) and Limit of Stability (LOS), evaluating motor control ability, were used in this study. ${ }^{19,20}$

The SMART Balance Master ${ }^{\circledR}$ system consists of two force platforms, a screen, and a main body of the computer. Subjects take off their shoes and stand with both legs on the center of two plates, then stare at the front monitor with folded arms. A preliminary trial was conducted in order to practice the test posture before starting the test.

SOT measures subjects' postural stability, how much they are swaying forward or backward from the center of gravity, while they maintain a standing posture for 20 seconds under each of 6 conditions, with a fixed/swaying force platform, open/closed eyes, and a fixed/moving screen. Condition 1 was a fixed force platform and a fixed screen, with open eyes; Condition 2 was a fixed 
force platform and a fixed screen, with closed eyes; Condition 3 a was fixed force platform and a moving screen, with open eyes; Condition 4 was a swaying force platform and a moving screen, with open eyes; Condition 5 was a swaying force platform and a fixed screen, with closed eyes; and Condition 6 was a swaying force platform and a moving screen with open eyes (Table 1, Fig. 2). Here, the COG sway was measured under three trials of each condition, and the measured sway was expressed in terms of an Equilibrium score (EQ).

The LOS test for motor control ability evaluation is a program to assess subjects' abilities to move their COG to fixed target points in a total of 8 directions: front, back, right, left, and each diagonal direction with five parameters of reaction time, movement velocity, directional control, endpoint excursion, and maximum excursion.

The evaluations above were conducted for both groups sequentially and were completed within 2 hours individuals had been using a computer. In statistical analysis, to compare mean results of neck angle, center of gravity, and balancing ability between groups, an independent ttest was used. To determine the effects of the severity of a forward head posture and the center of gravity on balancing ability, Pearson's correlation method was employed. The statistical significance was established at $\mathrm{p}<0.05$.

\section{RESULTS}

\section{General characteristics of study population}

In the experimental group, the mean age of participants was 34.9 years, their daily mean hours using a computer was 6.4 , and the mean service years in a computer-related occupation was 11.5 years; in the control group, the mean age of participants was 35.2 years, and their daily mean hours using a computer totaled fewer than 1 . Mean

Table 2. General Characteristics of Participants

\begin{tabular}{lcc}
\hline & $\begin{array}{c}\text { Group I } \\
(\mathbf{n = 3 0 )}\end{array}$ & $\begin{array}{c}\text { Group II } \\
(\mathbf{n = 3 0 )}\end{array}$ \\
\hline Age (years) & $34.9 \pm 2.1$ & $35.2 \pm 2.1$ \\
Sex & & \\
Male : Female & $22: 8$ & $21: 9$ \\
Height (cm) & $173.6 \pm 6.2$ & $173.4 \pm 6.7$ \\
Body weight (kg) & $72.5 \pm 5.1$ & $70.5 \pm 4.9$ \\
Computer (hours/day) & $6.5 \pm 0.4$ & $0.8 \pm 0.7$ \\
\hline
\end{tabular}

Values are mean \pm standard deviation height was $173.6 \pm 6.2 \mathrm{~cm}$ and $173 \pm 6.7 \mathrm{~cm}$ respectively. Mean weight was $71.2 \pm 2.4 \mathrm{~kg}$ and $72.3 \pm 4.5 \mathrm{~kg}$ respectively (Table 2).

\section{Comparison of results from experiment and control groups}

After taking pictures from a lateral position during computer operation, the monitor-facing angle (Angle A) and the angle of the cervical curve from the $\mathrm{C} 7$ spinous process (Angle B) were determined. By subtracting Angle A from Angle B, the severity of the forward head posture was quantified, showing that the experiment group had statistically significant low values for Angle B minus Angle A (Table 3 ).

Using a foot pressure measurement system, the center of gravity (COG) was measured in static and dynamic status. This showed that the experimental group had their COG in a relatively anterior location compared to the control group, and the difference was statistically significant (Table 3).

In the SOT assessing balancing ability, the experimental group had significantly lower equilibrium scores (EQ) in Conditions 5 and 6, compared to the control group (Table 3). Also, in the LOS test, the experiment group had significantly lower scores for movement velocity, maximum excursion, and endpoint excursion (Table 4).

Table 3. The Comparison of Head and Neck Posture, COG, SOT (EQ Score)

\begin{tabular}{lll}
\hline & Group I & Group II \\
\hline Angle A & $20.6 \pm 5.6$ & $18.9 \pm 1.8$ \\
Angle B & $48.9 \pm 4.3^{*}$ & $51.9 \pm 5.5$ \\
Angle B-A & $28.2 \pm 8.3^{*}$ & $32.9 \pm 6.0$ \\
COG (Static) & $53.6 \pm 5.9^{*}$ & $50.5 \pm 5.3$ \\
COG (Dynamic) & $58.5 \pm 3.1^{*}$ & $53.7 \pm 4.6$ \\
Condition1 (EQ score) & $95.1 \pm 1.3$ & $95.2 \pm 1.4$ \\
Condition2 (EQ score) & $92.2 \pm 2.0$ & $92.8 \pm 1.7$ \\
Condition3 (EQ score) & $87.8 \pm 4.2$ & $89.1 \pm 3.8$ \\
Condition4 (EQ score) & $83.9 \pm 4.6$ & $84.9 \pm 3.0$ \\
Condition5 (EQ score) & $72.6 \pm 5.9^{*}$ & $76.3 \pm 2.8$ \\
Condition6 (EQ score) & $69.0 \pm 7.2^{*}$ & $72.8 \pm 3.9$ \\
\hline
\end{tabular}

Values are mean \pm standard deviation

COG: Center of gravity, SOT: Sensory organization test, EQ score: Equilibrium score

${ }^{*} \mathrm{p}<0.05$ by Independent t-test: compared to Group II 
Assessing the interactions among forward head posture, COG, and balancing ability

The severity of forward head posture was meaningfully associated with changed COG in the anterior direction in static and dynamic conditions. In parameters to assess balancing ability, SOT condition 6 was seen to be related to the severity of a forward head posture $(\mathrm{p}<0.05)$. In addition, while a changed COG in the anterior direction under static conditions was associated with balancing ability in SOT condition 6 only $(\mathrm{p}<0.05)$, the anterior movement of COG under dynamic conditions showed a meaningful relationship with balancing ability in conditions 5 and 6 (Table 5).

\section{DISCUSSION}

The forward head syndrome is one of several VDT (Visual Display Terminals) syndromes and is commonly found in computer users who work in a consistent posture for extended periods of time. To assess head and neck postures of subjects, lateral pictures were taken and then the angle of cervical curve and the monitor-facing angles were measured. This method has been frequently

Table 4. The Comparison of LOS

\begin{tabular}{lccrrr}
\hline & \multicolumn{2}{c}{ Group I } & & \multicolumn{2}{c}{ Group II } \\
\cline { 2 - 3 } \cline { 5 - 6 } & Forward & Backward & & Forward & Backward \\
\hline RT (s) & $0.94 \pm 0.38$ & $0.82 \pm 0.25$ & & $0.91 \pm 0.30$ & $0.87 \pm 0.24$ \\
MVL (deg/s) & $3.33 \pm 1.00^{*}$ & $3 \pm 0.91^{*}$ & & $4 \pm 0.98$ & $3.73 \pm 0.97$ \\
EPE (\%) & $71 \pm 9.23^{*}$ & $51.1 \pm 9.6^{*}$ & & $79.4 \pm 9.43$ & $59.1 \pm 9.34$ \\
MXE (\%) & $82.3 \pm 0.25^{*}$ & $65.6 \pm 7.73^{*}$ & & $89.7 \pm 6.48$ & $72 \pm 8.00$ \\
DCL (\%) & $78.2 \pm 9.05$ & $67.2 \pm 8.16$ & & $81.7 \pm 4.96$ & $68.1 \pm 7.21$ \\
\hline
\end{tabular}

Values are mean \pm standard deviation

LOS: Limit of stability, RT: Reaction time, MVL: Movement velocity, EPE: Endpoint excursion, MXE: Maximum excursion, DCL: Directional control

${ }^{*} \mathrm{p}<0.05$ by Independent $\mathrm{t}$-test: compared to Group II utilized in previous studies, including studies by Sliva and Harrison. ${ }^{11,16,17}$ Heavy computer users, who use a computer for at least 6 hours daily over a 10 year period, showed statistically reduced values in Angle (B-A), compared to the control group. A more severely protruded head causes a more decreased angle of cervical flextion (Angle B) and an increased staring angle (Angle A); thus, Angle (B-A) is reduced. Due to the protruded head toward the anterior direction and an increased staring angle (Angle A), the upper cervical vertebrae comes under extension moment. This causes a transition of COG to the anterior direction under both static and dynamic conditions.

Maintaining balance is affected by many factors, including vision, proprioception, inner ear and vestibular function, cerebellum function, feet location on a surface, difference in length of both legs, muscular strength of legs, medication, aging, heartbeat or respiration, various musculoskeletal disorders, and hearing sense..$^{21}$ This study showed that heavy computer user had significantly decreased ability to control posture and mobility, compared to the control group, even though they do not necessarily complain of severe pain caused by musculoskeletal disorders such as myofascial pain syndrome or herniated disc.

The computerized dynamic posturography (CDP) used to evaluate balance maintenance was developed by Nashner et al. in 1986, and many studies have been conducted using this. Although some have reported negative aspects in clinical application, the CDP, in general, is useful for selective and comprehensive evaluation of sensory and motor systems. It is unlike conventional methods and thus has been widely used in clinical settings. ${ }^{19,20}$

This study evaluated subjects' abilities to control body posture and mobility, using Balance Master ${ }^{\circledR}$ system. SOT, a program evaluating how well subjects can control their posture under 6 conditions, showed no significant

Table 5. Correlation between Head and Neck Posture, COG and Postural Balance

\begin{tabular}{lccccccc}
\hline & B-A & SOT1 & SOT2 & SOT3 & SOT4 & SOT5 & SOT6 \\
\hline B-A & & .19 & .04 & .15 & .24 & .28 & $.57^{*}$ \\
COG (Static) & $-.62^{*}$ & .02 & .03 & -.04 & -.12 & -.33 & $-.36^{*}$ \\
COG (Dynamic) & $-.42^{*}$ & .03 & -.05 & -.14 & -.16 & $-.36^{*}$ &.$- .70^{*}$ \\
\hline
\end{tabular}

Values are Pearson correlation coefficient (R)

B-A: Angle B-Angle A, SOT: Sensory organization test, COG: Center of gravity

${ }^{*} \mathrm{p}<0.05$ by Pearson correlation coefficient 
difference between the experimental and control groups in conditions 1, 2, 3, and 4; however, in conditions 5 and 6 , decreased balancing ability was found in the experimental group, compared to the control, with EQ that was significantly related to the severity of a forward head posture and anterior movement of COG. People maintain their body balance with their hips and ankle joints, by obtaining information about balance through somatic sensory, visual, and vestibular systems, ${ }^{21-23}$ then organizing the information with a composition process in the central nerve system. For the critical information for balancing obtained from somatic sensory, visual, and vestibular systems, condition 5 utilized a swaying platform and closed eyes to block visual clues and induce confusion of somatic sensory, in order to examine balancing ability achieved by the vestibular system only. Unlike condition 5 , condition 6 , in spite of open eyes, offers false visual information with a moving screen in the forward and backward positions. Such sensory that causes confusion, instead of helping recognition for equilibrium, is called confusion sensory. ${ }^{20}$

According to Dornan et al., ${ }^{23}$ visual recognition is a key factor in maintaining posture and balance in the static condition. Therefore, although computer-based workers have reduced balancing ability, as long as the level is not significant, their slight defect is not revealed under conditions in which they can use their normal somatic sensory, visual, and vestibular systems for balancing, such as in condition 1, 2 and 3 of the static condition with a fixed platform, and condition 4, with a swaying platform but with open eyes. However, when critical information for balancing is blocked, like in conditions 5 and 6 (in other words, under situations where individuals cannot maintain balance easily), this significantly reduces posture control ability, compared to the control group.

In the LOS test for motor control ability assessment, the experimental group showed reduced motor control ability, with significantly lower scores in movement velocity, maximum excursion, and endpoint excursion, with the exceptions of reaction time and directional control. The center of gravity determined by Gait View ${ }^{\circledR}$ was moved forward, with results of $53.6 \%$ and $58.5 \%$ in static and dynamic condition respectively. The experimental group showed the larger change in COG to the anterior direction compared with the control group (50.5\% and 53.7\%, respectively). Forward COG causes extended stretching of ankle joints to maintain body balance, and the stretched ankle joints restrict movement of knee and hip joints. ${ }^{24}$ Therefore, the experiment group has more restricted movement capacity of the lower limb joints than the control group, which makes it difficult to control velocity in anteroposterior body sway and also to lean the body to the maximum extent while maintaining balance. Reaction time involves measuring time of the first body movement after starting the test, which shows how quickly the body organizes input sensory information in the central nerve and shows an output body reaction. As gender and age were controlled for, and other disorders that may affect balance were excluded, there were no significant differences in reaction times. In spite of the exaggeratedly anterior location of COG with a protruded head, absence of body asymmetry in the right and left sides contribute to maintaining the ability to control direction and movement in the other direction; no significant intergroup difference was found.

In previous studies, Ming et al. ${ }^{25}$ explored repetitive use of computers for an extended period of time and the neck and shoulder pain that can be related to, and caused by, computer use (NSPRCU), Poole et al. ${ }^{15}$ reported that patients with neck pain showed decreased ability in maintaining balance and gait. However, this study revealed that heavy computer users have relatively protruded heads and their COG were shifted anteriorly to maintain balance. In quantification of their balancing abilities, these individuals had posture imbalance and relatively reduced motor control ability.

Screening the participants revealed that heavy computer users were mostly office workers, and their usual exercise levels were not included; this may have resulted in a potential screening-related error. Moreover, although physical and radiological examinations were conducted in order to exclude cervical disorders that may affect balancing ability, precise tests such as an MRI were not used; it is a limit of the study that it cannot definitely rule out the possible presence of musculoskeletal disorder.

\section{CONCLUSION}

This study indicates that heavy computer users tend to have a more protruded head than the control group, and the foot pressure measurement system and Balance Mas$\operatorname{ter}^{\circledR}$ system confirmed that their COG was moved forward 
and their balancing ability was reduced. Therefore, this study is expected to support existing data and further emphasize the importance of good posture education and stretching exercises whilst using a computer and common VDTs such as Smart phones. There remains a need for further study in order to understand the effects of posture education on the incidence of musculoskeletal complications in heavy computer users.

\section{REFERENCES}

1. Martin L, Scannell GF. Working safely with video display terminals. USA, OSHA, 1997, 4

2. AMA. Health effects of video display terminals. Council on Scientific Affairs. JAMA 1987; 257: 1508-1512

3. Rempel DM, Harrison RJ, Barnhart S. Work-related cumulative trauma disorders of the upper extremity. JAMA 1992; 267: 838-842

4. Picavet HS, Schouten JS. Musculoskeletal pain in the Netherlands: prevalences, consequences and risk groups, the DMC(3)-study. Pain 2003; 102: 167-178

5. Kim DQ, Cho SH, Han TR, Kwon HJ, Ha M, Paik NJ. The effect of VDT work on work-related musculoskeletal disorder. Korean J Occup Environ Med 1998; 10: 524-533

6. Cho WH, Lee WY, Choi HK. An investigation on the biomechanical effects of turtle neck syndrome through EMG analysis. KSPE 2008; 1: 195-196

7. Yoo WG, YI CH, Jeon HS, Cynn HS, Choi HS. Effects of the height of ball-baskrest on head and shoulder posture and trunk muscle activity in VDT workers. Ind Health 2008; 46: 289-297

8. Szeto GP, Straker L, Raine S. A field comparison of neck and shoulder postures in symptomatic and asymptomatic office workers. Appl Ergon 2002; 33: 75-84

9. Moore MK. Upper crossed syndrome and its relationship to cervicogenic headache. J Manipulative Physiol Ther 2004; 27: 414-420

10. Burgess-Limerick R, Plooy A, Ankrum DR. The effect of imposed and self-selected computer monitor height on posture and gaze angle. Clin Biomech 1998; 13: 584-592

11. Silva AG, Punt TD, Sharples P, Vilas-Boas JP, Johnson MI. Head posture and neck pain of chronic nontraumatic origin: a comparison between patients and pain-free persons. Arch Phys Med Rehabil 2009; 90: 669-674

12. Griegel-Morris P, Larson K, Mueller-Klaus K, Oatis CA. Incidence of common postural abnormalities in the cervical, shoulder and thoracic regions and their association with pain in two age groups of healthy subjects. Phys Ther 1992; 72: 425-431

13. Chester JB Jr. Whiplash, postural control and the inner ear. Spine 1991; 7: 716-720

14. Barrett DS, Cobb AG, Bentley G. Joint proprioception in normal, osteoarthritic and replaced knees. J Bone Joint Surg Br 1991; 73: 53-56

15. Poole E, Treleaven J, Jull G. The influence of neck pain on balance and gait parameters in community-dwelling elders. Man Ther 2008; 13: 317-324

16. Harrison AL, Barry-Greb T, Wojtowicz G. Clinical measurement of head and shoulder posture variables. J Orthop Sports Phys Ther 1996; 23: 353-361

17. Visscher CM, De Boer W, Lobbezoo F, Habets LL, Naeije M. Is there a relationship between head posture and craniomandibular pain? J Oral Rehabil 2002; 29: 1030-1036

18. Park BJ, Park SB, Park JW, Lee SJ, Lee KH. The effects of physical factors on anteroposterior, intersical weight-bearing pattern and stance phase in normal adults. J Korean Acad Rehab Med 2008; 32: 576-581

19. Goh EK. Diagnostic significance of dynamic posturography. J Pusan Med 1991; 31: 343-350

20. Goh EK. Clinical application of computerized dynamic posturography. Res Vestibul Sci 2005; 4: 107-118

21. Di Fabio RP. Sensitivity and specificity of platform posturography for identifying patients with vestibular dysfunction. Phys Ther 1995; 75: 290-305

22. Umphred DA. Neurological rehabilitation, 4th ed, St Louis, Mosby, 2001, 616-660

23. Dornan J, Fernie GR, Holliday PJ. Visual input: its importance in the control of postural sway. Arch Phys Med Rehabil 1978; 59: 586-591

24. Vernazza S, Alexandrov A, Massion J. Is the center of gravity controlled during upper trunk movements. Neuroscience Lett 1996; 206: 77-80

25. Ming Z, Narhi M, Siivola J. Neck and shoulder pain related to computer use. Pathophysiology 2004; 11: 5156 